\title{
Ionic Desorption in Valence- and Core- Excited Polymers: Poly(vinyl Chloride) and Poly(vinylidene Chloride)
}

\author{
M. L. M. Rocco, G. S. Faraudo, F. C. Pontes, G. G. B. de Souza, \\ Instituto de Química, Universidade Federal do Rio de Janeiro, \\ Cidade Universitária, Ilha do Fundão, 21949-900, Rio de Janeiro, RJ, Brazil \\ R. R. Pinho, \\ Departamento de Física-ICE, Universidade Federal de Juiz de Fora, \\ Campus Universitário, 36036-330, Juiz de Fora, MG, Brazil \\ and M. Ferreira \\ PEMM/COPPE, Universidade Federal do Rio de Janeiro, \\ Cidade Universitária, Ilha do Fundão, 21941-972, Rio de Janeiro, RJ, Brazil
}

Received on 29 July, 2005

\begin{abstract}
Photon stimulated ion desorption (PSID) studies have been performed in poly(vinyl chloride) (PVC) and poly(vinylidene chloride) (PVDC) using synchrotron radiation, covering from valence to core electron $(\mathrm{Cl} 2 \mathrm{p}$ and C 1s) energy ranges. Data acquisition was performed at the TGM beam line from the Brazilian Synchrotron Light Source (LNLS), operating in a multi-bunch mode and using a time-of-flight mass spectrometer (TOFMS). A new pulsed system developed uses as a trigger for the TOF-MS experiments the pulsed extraction high voltage applied to the sample. Ionic desorption from PVC and PVDC shows strong selectivity in the formation of chlorine ions around the $\mathrm{Cl} 2 \mathrm{p}$-edge while very similar fragmentation patterns are observed for the other energies studied.
\end{abstract}

Keywords: Photon stimulated ion desorption (PSID); Polymers; Synchrotron radiation

\section{INTRODUCTION}

The availability of intense and tunable photon sources (synchrotron radiation) has made possible the analysis of the ionic emission from surfaces as a continuous function of the photon energy. The study of the interaction of high-energy photons with polymers is a topic of increasing interest mainly due to their use in many technological applications, like for instance in areas such as microfabrication in which polymers are routinely used as resists $[1,2]$. In addition, there is a special interest in the study of surface photochemistry by core level photoexcitation experiments using synchrotron radiation because inner-shell processes show element and site selectivity. One attractive topic in this context is the capability of selectively breaking a chemical bond [3]. It is believed that the main process governing ion desorption is the Auger process, which can be described in a simplified form as a relaxation process following excitation (resonant Auger) or ionization (normal Auger) of a core electron. Several examples are now known in which, following the ionization or excitation of an inner-shell electron and the corresponding relaxation through the Auger process, selective fragmentation is observed around the atom to which the inner-shell electron is closely associated [3-9]. In this paper we present photon stimulated ion desorption (PSID) spectra for poly(vinyl chloride) (PVC) and poly(vinylidene chloride) (PVDC), irradiated in the 21.21 to $310 \mathrm{eV}$ photon energy range. This broad energy interval allows for a general observation of the photon energy dependence of the fragmentation pattern associated with single and double (multiple) ionization processes as well as Auger-assisted double (or even triple) ionization channels. The measurements were performed at the Brazilian Synchrotron Light Source (LNLS), using a time-of-flight mass spectrometer (TOF-MS). The mass scale calibration procedure used in ESID studies of PMMA [10] was also successfully employed in the present PSID studies. The results show new evidence for site-specificity in the ionic desorption from PVC and PVDC, irradiated at the chlorine $2 \mathrm{p}$-edge.

\section{EXPERIMENTAL}

Ionic desorption experiments were performed at the TGM beamline $(12-310 \mathrm{eV})$ from the Brazilian Synchrotron Light Source (LNLS), located at the city of Campinas, São Paulo. The experimental setup includes a sample manipulator and a time-of-flight mass spectrometer (TOF-MS), which are housed in an ultra-high vacuum chamber (UHV) with a base pressure of about $10^{-9}$ Torr. The TOF-MS employed in the present work has been described in detail elsewhere [11]. Basically, it consists of an electrostatic ion extraction system, a drift tube and a pair of microchannel plate (MCP) detectors, disposed in the chevron configuration. After extraction, positive ions travel through three metallic grids (each of which with a nominal transmission of $90 \%$ ), before reaching the MCP. Ion optics simulation using the SIMION program [12] shows that the use of a collimating electrostatic lens, which was incorporated in the TOF-MS, allows for an efficiency close to $100 \%$ in the extraction of ions with up to 40 eV kinetic energy. In order to perform PSID studies using 
synchrotron radiation from the Brazilian storage ring, which is normally operated in a multi-bunch mode, a pulsed high voltage applied to the sample was used as a trigger for the TOF-MS experiments [13]. In this pulsed system, the start signals to the TDC (time-to-digital converter) were provided by a signal associated to the rising edge of the extraction pulse, usually of $+250 \mathrm{~V}$, applied to the solid sample. Pulse widths from $300 \mathrm{~ns}$ to $1.5 \mu$ s with a repetition interval of $150 \mu$ s, provided by a homemade pulse generator, were employed. The output signal of the detector was processed by a standard pulse counting system and used to provide a stop signal to the TDC. The incident beam makes a $60^{\circ}$ angle with respect to the normal to the sample, which in its turn was about $25 \mathrm{~mm}$ away from the first grid of the TOF spectrometer. Thin films of PVC and PVDC were prepared by the spin casting technique. No additional cleaning procedure was adopted. The samples were attached to an aluminum support, which was mounted onto the X-Y-Z sample manipulator. For a precise calibration of the mass spectra we have used a new calibration procedure previously employed in ESID studies of PMMA [10]. Basically, it consists of introducing a small amount of a noble gas into the vacuum chamber, raising the pressure from $10^{-9}$ to about $10^{-7}$ Torr and recording a TOF spectrum of the desorbing ions emanating simultaneously from the atomic gas and from the polymer surface. In our case, argon gas was used, thus allowing for an accurate mass scale calibration.

\section{RESULTS AND DISCUSSION}

Photon stimulated ion desorption (PSID) spectra for PVC and PVDC were measured at different photon energies covering the valence region as well as the chlorine $2 \mathrm{p}$ and carbon $1 \mathrm{~s}$ absorption edges. In all measured spectra $\mathrm{H}^{+}$could not be observed, due to an instrumental noise associated with the pulse generator employed. Spectra were also obtained at $21.21 \mathrm{eV}$ but no ions were observed, which is probably connected to a fast and efficient reneutralization of the ions at the polymer surface. PSID spectra for PVC measured at 110, 200, 210 and $250 \mathrm{eV}$ photon energy, and covering a broad mass range (up to 140 a.m.u.) are presented in Fig. 1. Spectra at 35 and 40.8 $\mathrm{eV}$ were also obtained [14] but they show the same fragmentation patterns as the spectrum at $110 \mathrm{eV}$ presented in Fig. 1. At this low covered photon energy range (up to $110 \mathrm{eV}$ ), the observed positively charged fragments could only be associated with the direct ionization (single, double, or even triple) of valence-shell electrons. As the energy increases, core electron excitation and ionization processes can be reached.

In all spectra three main groups of ions are observed. The main contributions to the first group of ions are centered at $\mathrm{m} / \mathrm{q}=27$ a.m.u. $\left(\mathrm{C}_{2} \mathrm{H}_{3}^{+}\right)$and $\mathrm{m} / \mathrm{q}=29$ a.m.u. $\left(\mathrm{C}_{2} \mathrm{H}_{5}^{+}\right)$. Both fragments are related to a $\mathrm{C}-\mathrm{Cl}$ bond breaking process in which neutral $\mathrm{Cl}$ radicals are ejected. The $\mathrm{C}_{2} \mathrm{H}_{5}^{+}$cation is the signature of a rearrangement reaction. This group of ions shows a close correspondence to the mass spectrum of chloroethane, in which the cations $\mathrm{C}_{2} \mathrm{H}_{n}^{+}(\mathrm{n}=2$ - 6) are clearly observed [15]. The second group shows two intense peaks at $\mathrm{m} / \mathrm{q}=41$ a.m.u. $\left(\mathrm{C}_{3} \mathrm{H}_{5}^{+}\right)$and $\mathrm{m} / \mathrm{q}=44$ a.m.u. $\left(\mathrm{C}_{3} \mathrm{H}_{8}^{+}\right)$. Again
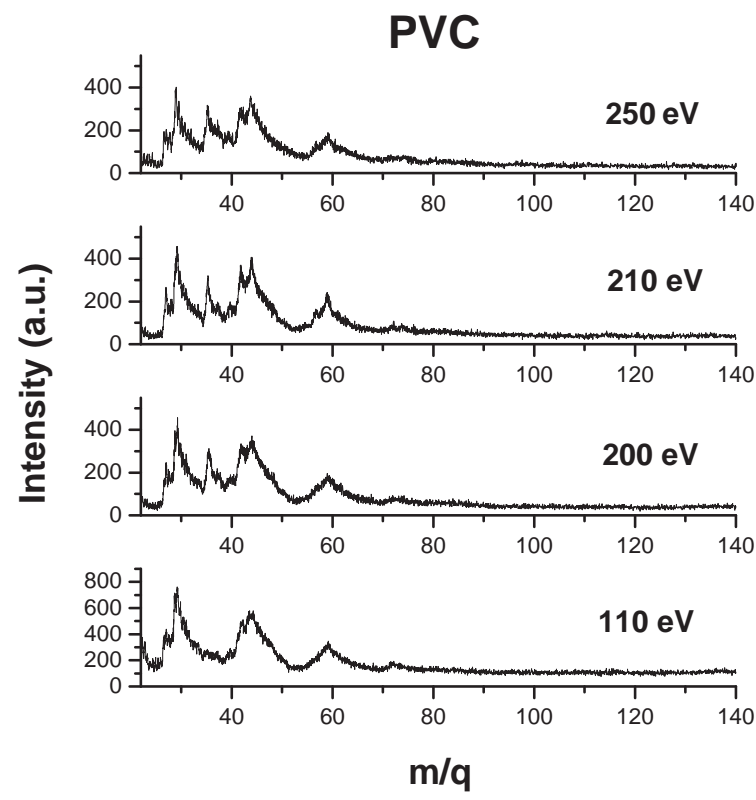

FIG. 1: Ionic desorption mass spectra of PVC induced by 110, 200, 210 and $250 \mathrm{eV}$ photon energies.

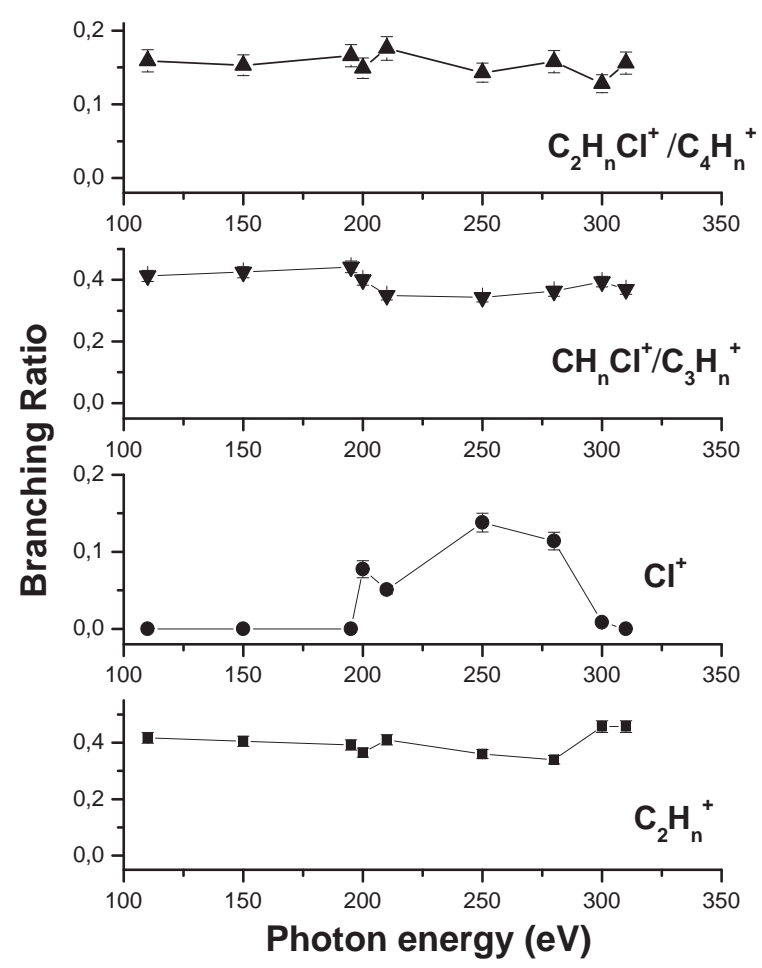

FIG. 2: Branching ratios for different fragments of PVC. 


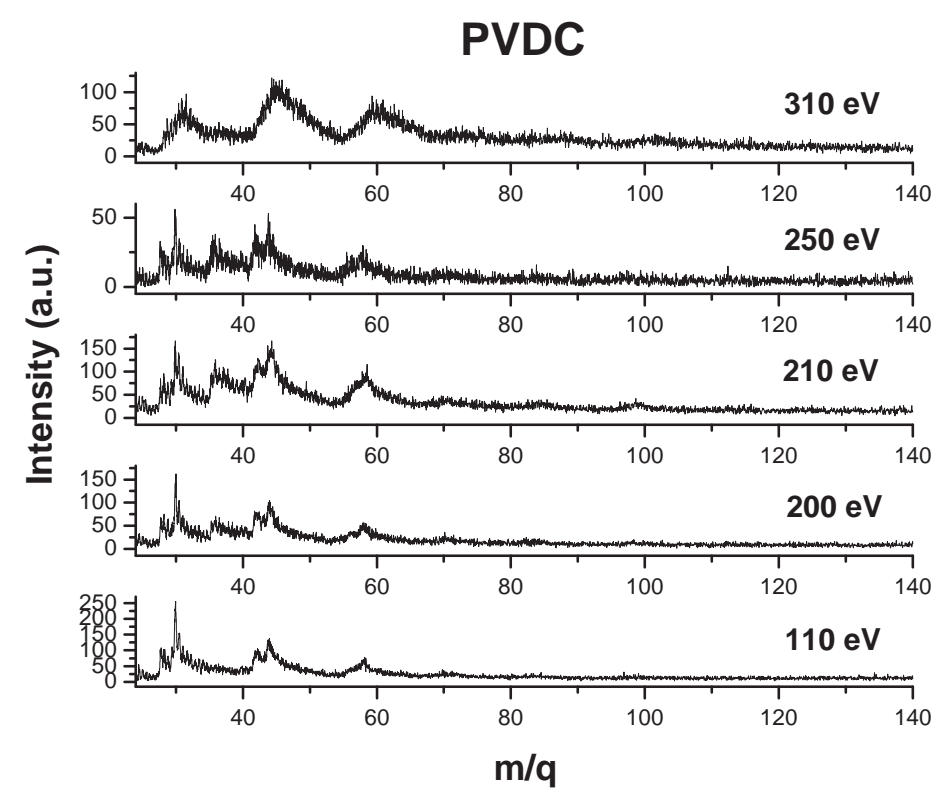

FIG. 3: Ionic desorption mass spectra of PVDC induced by 110, 200, 210, 250 and $310 \mathrm{eV}$ photon energies.

the fragments are related to the loss of chlorine atoms and rearrangement reactions. The enlargement of this band could be associated to $\mathrm{CH}_{n} \mathrm{Cl}^{+}$contributions, also expected for the chloroethane molecule [15]. The third main group is roughly centered at $\mathrm{m} / \mathrm{q}=59$ a.m.u. This large band can in principle be assigned to a mixture of $\mathrm{C}_{2} \mathrm{H}_{n} \mathrm{Cl}^{+}$and $\mathrm{C}_{4} \mathrm{H}_{n}^{+}$fragments. Two additional weaker structures can also be seen at $\mathrm{m} / \mathrm{q}=$ 40 a.m.u. $\left(\mathrm{C}_{3} \mathrm{H}_{4}^{+}\right)$and $\mathrm{m} / \mathrm{q}=72$ a.m.u. $\left(\mathrm{C}_{5} \mathrm{H}_{12}^{+}\right.$or $\left.\mathrm{C}_{3} \mathrm{HCl}^{+}\right)$. Ions with larger $\mathrm{m} / \mathrm{q}$ values were not detected. In addition, in the spectra measured around the $\mathrm{Cl} 2 \mathrm{p}$-edge, a dramatic change in the fragmentation pattern was observed. New and intense peaks are observed at $\mathrm{m} / \mathrm{q}=35$ and 37 , corresponding to the formation of the $\mathrm{Cl}^{+}$cation. The isotopic abundance of 3:1, expected for ${ }^{35} \mathrm{Cl}^{+}$and ${ }^{37} \mathrm{Cl}^{+}$, can be clearly observed, particularly at the mass spectra measured at 200 and $210 \mathrm{eV}$. The excitation and ionization of core electrons are followed by two efficient relaxation processes namely, resonant Auger and normal Auger. In resonant Auger, a given core electron is initially promoted to an unoccupied valence orbital. The excited electron then either remains as a spectator (Spectator Auger) or participates (Participator Auger) in the subsequent relaxation mechanism by which the initial core hole is filled by a valence-shell electron. In normal Auger, the first step is the core electron ionization. A valence-shell electron then fills the hole and another electron is ejected, the so-called Auger electron. As a result, two valence-shell electrons are ejected and a doubly-charged species is formed. Most doubly-charged ions are unstable and as a result they dissociate into two fast ions (Coulomb explosion). Fast ions have a higher chance of overcoming the surface barrier and reneutralization processes.

Branching ratios, i.e. the area of a given peak or group of peaks divided by the total area of the spectrum, were deter- mined for the main three groups of fragments plus the chlorine cation, and the results are presented as a function of the photon energy in Figure 2. The branching ratios corresponding to the $\mathrm{C}_{2} \mathrm{H}_{n}^{+}, \mathrm{CH}_{n} \mathrm{Cl}^{+} / \mathrm{C}_{3} \mathrm{H}_{n}^{+}$and $\mathrm{C}_{2} \mathrm{H}_{n} \mathrm{Cl}^{+} / \mathrm{C}_{4} \mathrm{H}_{n}^{+}$fragments are basically energy-independent in the covered photon energy range. The chlorine ion, on the other hand, shows a definite resonating behavior, as the relative probability of formation of this cation is clearly enhanced along the $\mathrm{Cl} 2 \mathrm{p}$ excitation energy range. This feature, which we consider to be associated with resonant and normal Auger processes along the $\mathrm{Cl} 2 \mathrm{p}$-edge, is discussed in more detail in an additional publication [16]. PSID spectra for PVDC are shown in Figure 3 covering a broad photon energy as well as mass interval. Similar fragmentation patterns as a function of the photon energy can be observed for the PVDC polymer when compared to PVC. Three main groups of fragments dominate the PSID spectra except for the $\mathrm{Cl} 2 \mathrm{p}$ absorption edge. Again it is possible to observe strong selectivity in the formation of chlorine ions around the $\mathrm{Cl} 2 \mathrm{p}$-edge while very similar fragmentation patterns are observed for the other energies studied.

\section{CONCLUSIONS}

Photon stimulated ion desorption (PSID) studies have been performed in PVC and PVDC using synchrotron radiation at the valence region as well as at the chlorine $2 \mathrm{p}$ - and carbon $1 \mathrm{~s}-$ edges. The experimental results were obtained using the TGM beam line from the Brazilian Synchrotron Light Source. A time-of-flight mass spectrometer (TOF-MS) and a pulsed system were used to obtain the PSID data. Three main groups of ions are seen to dominate the mass spectra at the ener- 
gies investigated. However, strong evidence for selective bond breaking around the chlorine $2 \mathrm{p}$-edge was observed for both polymers. would like to thank CNPq and FAPERJ for financial support. Authors would also like to thank Prof. Arnaldo N. de Brito as well as the VUV-Group from LNLS for technical assistance.

\section{Acknowledgments}

Research partially supported by LNLS - National Synchrotron Light Laboratory, Brazil. M.L.M.R. and G.G.B.S.

[1] O. Wollersheim, H. Zumaqu, J. Hormes, D. Kadereit, J. Langen, L. Häußling, P. Hoessel, and G. Hoffmann, Nucl. Instrum. Meth. B 97, 273 (1995).

[2] Y. Zhang, T. Katoh, Jpn. J. Appl. Phys. 35, L186 (1996).

[3] Y. Baba, Low Temp. Phys. 29, 228 (2003).

[4] M. C. K. Tinone, K. Tanaka, J. Maruyama, N. Ueno, M. Imamura, and N. Matsubayashi, J. Chem. Phys. 100, 5988 (1994).

[5] K. Tanaka, M. C. K. Tinone, H. Ikeura, T. Sekiguchi, and T. Sekitani, Rev. Sci. Instrum. 66, 1474 (1995).

[6] N. Ueno, K. Tanaka, Jpn. J. Appl. Phys. 36, 7605 (1997).

[7] K. Fujii, H. Tomimoto, K. Isshiki, M. Tooyama, T. Sekitani, and K. Tanaka, Jpn. J. Appl. Phys. 38, 321 (1999).

[8] T. Sekitani, E. Ikenaga, K. Fujii, K. Mase, N. Ueno, and K. Tanaka, J. Electron Spectrosc. Relat. Phenom. 101-103, 135 (1999).

[9] S. Wada, R. Sumii, K. Isari, S. Waki, E.O. Sako, T. Sekiguchi, T. Sekitani, and K. Tanaka, Surf. Sci. 528, 242 (2003).
[10] M. L. R. Rocco, G. G. B. de Souza, and D. E. Weibel, Rev. Sci. Instrum. 72, 2827 (2001).

[11] J. B. Maciel, E. Morikawa, and G. G. B. de Souza, Proceedings Synchrotron Radiation Instrumentation, National Conference, AIP Conf. Proc., 417 (1997).

[12] D. A. Dahl, Simion 3D Version 6.0 User's Manual, Idaho National Engineering Laboratory, Ion Source Software, 1995.

[13] M. L. M. Rocco, F. C. Pontes, G. S. Faraudo, and G. G. B. de Souza, Polym. Degrad. Stab. 88, 213 (2005).

[14] M. L. M. Rocco, G. S. Faraudo, R. R. Pinho, M. Ferreira, F. C. Pontes, and G. G. B. de Souza, J. Electron Spectrosc. Relat. Phenom. 141, 1 (2004).

[15] http://webbook.nist.gov/chemistry

[16] M. L. M. Rocco, G. S. Faraudo, F. C. Pontes, R. R. Pinho, M. Ferreira, and G. G. B. de Souza, Chem. Phys. Lett. 393, 213 (2004). 